\title{
Initial Experience with Robotic Surgical Exclusion of the Left Atrial Appendage
}

\author{
Nathan Smith, $M D^{1}$, Erin Harris², Benjamin Gallant ${ }^{2}$, Olatoye Olutola, MD ${ }^{3}$, Sanjay Samy \\ $M D^{1}$ and Walter Scott ${ }^{1}$
}

${ }^{1}$ Department of Cardiothoracic Surgery, Albany Medical Center, USA

${ }^{2}$ Albany Medical College, USA

${ }^{3}$ Department of General Surgery, Albany Medical Center, USA

\begin{abstract}
Introduction: Atrial fibrillation (AF) is a major modifiable risk factor associated with an increased risk of ischemic stroke. It is an independent predictor of mortality and is associated with poorer outcomes among stroke patients. AF affects approximately 2.7 million people with a stroke rate of $3.5 \%$ per year. Amongst individuals with non-valvular $\mathrm{AF}$ that experience embolic stokes, $90 \%$ arise from the left atrial appendage (LAA), and consequently left atrial appendage closure can be considered for patients unable to tolerate anticoagulation medications. Surgical exclusion of the LAA decreases the yearly risk of stroke to $0.7 \%$ when used in combination with a Maze procedure. While not as effective in preventing strokes as other anti-arrhythmic procedures such as a maze, epicardial exclusion via endovascular or epicardial techniques can be used to eliminate the thrombogenic, low-flow cavity of the left atrial appendage, theoretically reducing the stroke risk by $90 \%$. Epicardial clipping can be done through a sternotomy while performing a concomitant procedure, or via a minimally invasive approach using a video-assisted surgical or a robotic approach. We present here our initial institutional experience of epicardial clipping of the LAA using a robotic assisted thoracoscopic approach.
\end{abstract}

Results: The mean age was 75.9-years. The mean CHA2DS2VASC score was 4.3, and the mean HAS-BLED. score was 3.1. The mean postoperative length of stay was 2.2 days. Two cases were aborted due to an inability to achieve domain, and dense pleural adhesions, respectively. One patient developed acute pericarditis postoperatively that was easily treated with colchicine and pain medication. Another patient developed respiratory failure because of over-sedation. Finally, one of the patients developed hemorrhagic shock due to bleeding from pericardiophrenic artery, resulting in return to the operating room for median sternotomy.

Conclusion: Epicardial LAA clipping using a robotic approach shows promising results for high risk patients with AF, with fewer complications. Long term success in preventing stroke is still yet to be determined, but short-term results are encouraging and equivocal to the minimally invasive approach.

\section{Abbreviations}

AF: Atrial Fibrillation; CHF: Congestive Heart Failure; COPD: Chronic Obstructive Pulmonary Disease; IRB: Institutional Review Board; LAA: Left Atrial Appendage; LVEF: Left Ventricular Ejection Fraction; TIA: Transient Ischemic Attack

\section{Introduction}

Atrial fibrillation (AF) is the main cardiac abnormality associated with ischemic stroke. AF affects approximately 2.7 million people with an absolute stroke rate of 3.5\% per year [1]. Of the individuals with non-valvular AF that experience embolic stokes, $90 \%$ arise from the left atrial appendage (LAA) [2]. Since the 1940s, physicians and surgeons have studied methods to decrease the risk of embolic strokes due to AF [3]. These methods include anticoagulation and isolation of the LAA.

Anticoagulants, such as heparin and warfarin sodium, have long been the standard of care for patients with AF.
These medications, especially warfarin sodium, place patients at risk for bleeding complications such as hemorrhagic stroke, GI bleeding, intracranial hemorrhage, genitourinary bleeding, and spontaneous nasopharyngeal bleeding among other

*Corresponding author: Olatoye Olutola, MD, Department of General Surgery, Albany Medical Center, Albany, NY, USA

Accepted: May 03, 2021

Published online: May 05, 2021

Citation: Smith N, Harris E, Gallant B, et al. (2021) Initial Experience with Robotic Surgical Exclusion of the Left Atrial Appendage. J Cardiothorac Surg Ther 5(1):82-85 
complications. In a paper that studied the bleeding complications with Warfarin use from 1999-2003, it was associated with approximately 29,000 emergency department visits for bleeding complications per year. The risk of major bleeding in patients being treated with Warfarin is $10-16 \%$ [4]. Warfarin's therapeutic window of efficacy can also be compromised by dietary variation and differences in metabolism among individuals. Newer anticoagulants show promise for patients with $\mathrm{AF}$, but they are still at a risk of bleeding events. There are several scoring systems that predict the likelihood of stroke with $\mathrm{AF}$, the $\mathrm{CHA}_{2} \mathrm{DS}_{2} \mathrm{VASC}$ being one of the more widely used and verified [5-7]. In addition, the HAS-BLED score predicts the likelihood of bleeding while on anticoagulation [8-10].

Exclusion of the LAA is an essential maneuver in the Maze procedure for $A F$, which lowers the rate of stroke to $0.7 \%$ [11]. Traditional over-sewing of the LAA from inside the left atrium has been linked to a significant amount of recanalization [12]. Studies have shown that excision is superior to that of suture exclusion and stapler exclusion, resulting in more successful LAA closures for AF patients [13]. Incomplete closure of the LAA can produce an environment that is favorable to thrombogenesis and thus places the patient at an increased risk for future thromboembolic events [14]. An alternative technique is epicardial clipping which has been approved through sternotomy for permanent exclusion of the LAA $[15,16]$. Additional studies have shown that percutaneous occlusion of the LAA is effective and potentially superior to oral anticoagulants only in safely reducing the risk of stroke in patients with $A F$ [17]. Furthermore, new techniques with robotic LAA closure have shown significant superior and efficacy compared with that of suture exclusion and staple closure $[18,19]$.

Upon developing a robotic pulmonary and esophageal program at our institution, we began to approach the clipping of the left atrial appendage. We present our initial experience of epicardial clipping of the LAA using a robotic approach in patients with AF.

\section{Methods}

Our study was a retrospective observational case series design approved by the Institutional Review Board. Between July 2018 and September 2019, a total of 14 consecutive patients underwent left robotic assisted minimally invasive epicardial clipping. Our inclusion criteria were patients determined to be high risk for bleeding complications based on elevated HAS-BLED scores or actual severe bleeding episodes or other complications from anticoagulants. Contraindications included previous cardiac or pericardial operations due to adhesions between the heart and pericardium.

The procedure was performed in the following manner. After general anesthesia is induced, a single-lumen endotracheal tube and a left-sided bronchial blocker are placed. Appropriate lines (arterial line and two large bore intravenous lines or a central line, and a trans-esophageal echocardiography probe) are inserted. The clipping is performed with the patient in a supine position with a bump under the left hemi-thorax, exposing the entire left chest, along with the posterior axillary line. The left arm is tucked but padded and allowed to "fall" slightly posteriorly. The LAA is evaluated for thrombus, the left lung is isolated, and the left chest is entered at the mid-axillary line around the $5^{\text {th }}$ or $6^{\text {th }}$ intercostal space and gentle insufflation at $8 \mathrm{mmHg}$ begun. After confirming appropriately stable hemodynamics, a second robotic port is placed at the $7^{\text {th }}$ or $8^{\text {th }}$ intercostal space just anterior to the mid-axillary line, and a robotic force-bipolar arm is used for retraction. The third arm is placed at the posterior or mid-axillary line, depending on patient body habitus and intra-thoracic anatomy, and a robotic scissor with electrocautery is placed. The lung is retracted posteriorly, and the pericardium is opened from $6-7 \mathrm{~cm}$ below the pulmonary hilum (approximately $1-2 \mathrm{~cm}$ below the phrenic neurovascular bundle) and carried over the pulmonary veins until the left pulmonary artery became visible. Depending on the patient's individual anatomy, the pericardium is retracted with a silk stitch roughly 1-2 cm below the pulmonary hilum and either directly sutured to the chest wall or brought out anteriorly through the skin and snapped with a hemostat. The left atrial appendage base is measured, and the atrial clip was introduced into the left thorax via the inferior-most incision after undocking the robotic arm. Using the right robotic arm and a bedside assistant, the clip was gently maneuvered onto the left atrial appendage and clamped temporarily. Transesophageal echocardiography was used to evaluate the adequacy of appendage isolation, and if less than $1 \mathrm{~cm}$ of appendage was noted with no visible trabeculae, the clip was deployed. In the absence of those findings, the clip was released and re-maneuvered closer to the base and checked again. Once the clip is deployed, hemostasis is accomplished, and a single chest tube is placed through the inferior-most incision.

\section{Results}

Table 1 lists relevant patient characteristics. Six patients had paroxysmal AF, seven patients had persistent AF, and one patient had unspecified atrial fibrillation. The average age was 75.9 -years, with $50 \%$ above the age of 75 . A significant fraction of the patients suffered from various comorbid conditions. Most patients had a past medical history including $86 \%$ with hypertension, $64 \%$ with Coronary Artery Disease, $28 \%$ with diabetes and $21 \%$ with Congestive heart disease. Overall, the group of participants were moderate to high risk surgical candidates. The mean CHAD2VASC score was 4.3 and the mean HAS-BLED score was 3.1.

Table 2 contains indications for the procedure. Individual patients had various anticoagulation methods, including warfarin, factor Xa inhibitors and some not receiving any anticoagulation therapy. All anticoagulation therapy was discontinued either after a bleeding complication $(n=12)$ or before the procedure. A robotic assisted minimally invasive thoracoscopic approach was used in all patients. Two cases were aborted intra operatively; one was due to inability to obtain domain due to a small chest cavity and difficult lung isolation, and the other was secondary to extensive pleural and pericardial adhesions. All patients had successful exclusion of the LAA as defined by a residual stump less than $1 \mathrm{~cm}$ on transesophageal echocardiography. The mean postoperative length of stay was 2 days. 
Table 1: Demographics.

\begin{tabular}{|l|l|l|}
\hline Patient Characteristics & No. Patients & $\%$ \\
\hline Paroxysmal AF & 6 & 43 \\
\hline Persistent AF & 7 & 50 \\
\hline Unspecified AF & 1 & 7 \\
\hline Male & 4 & 28 \\
\hline > 75-years old & 7 & 50 \\
\hline 65-75-years old & 6 & 43 \\
\hline <65-years old & 1 & 7 \\
\hline Previous stroke/TIA & 1 & 7 \\
\hline Previous intracranial hemorrhage & 1 & 7 \\
\hline Hypertension & 12 & 86 \\
\hline Diabetes & 4 & 28 \\
\hline CHF or LVEF < 40\% & 3 & 21 \\
\hline Coronary Artery disease & 9 & 64 \\
\hline COPD & 1 & 7 \\
\hline Obesity & 3 & 21 \\
\hline Dyslipidemia & 12 & 86 \\
\hline Previous gastrointestinal bleeding & 8 & 57 \\
\hline Tobacco abuse & 5 & 36 \\
\hline Average HAS-BLED score & 3.1 & \\
\hline Average CHAD2S2-VASC score & 4.3 & \\
\hline & & 7 \\
\hline
\end{tabular}

Table 2: Indications.

\begin{tabular}{|l|l|l|}
\hline Indication & No. Patients & $\%$ \\
\hline Gastrointestinal bleed & 8 & 57 \\
\hline Hemorrhagic stroke & 1 & 7 \\
\hline Esophageal varices & 2 & 14 \\
\hline Skin Necrosis & 2 & 14 \\
\hline Radiation proctitis & 1 & 7 \\
\hline
\end{tabular}

Table 3: Complications.

\begin{tabular}{|l|l|l|}
\hline Complication & No. Patients & $\%$ \\
\hline Overall & 3 & 21 \\
\hline Death & 0 & 0 \\
\hline
\end{tabular}

Table 3 contains overall complications for the cohort of patients. Post-operatively, one patient was readmitted on postoperative day 2 for altered mental status and found to have to chest pain consistent with pericarditis which was treated with Colchicine. Another patient's postoperative course was complicated by respiratory failure from over-sedation requiring reintubation and transfer to the Intensive Care Unit. The patient was re-extubated and subsequently discharged on postoperative day 7. The last observed complication occurred after one of the patients became persistently hypotensive, with chest tube draining $1100 \mathrm{cc}$, refractory to blood products and pressors, resulting in return to operating room for control of pericardial bleeders with complete exci- sion of the left atrial appendage.

Follow-up was scheduled for 2-3 weeks after discharge. Twelve patients have been followed up post-operatively, and there have been no neurological or embolic events within one month of surgery. All patients have been discontinued using anticoagulation.

\section{Discussion}

The surgical approach to the LAA in patients with AF has undergone immense development since the 1940s. Initial methods included over-sewing to isolate the LAA. Numerous suturing practices have been described, including running simple, running double and purse string. Various open approaches also have been described, including intestinal stapling devices, excisional cutting and sewing and sequential ligating techniques. Percutaneous devices such as the Watch man's device has been showed to decrease the risk of stroke, however it requires a short period of anticoagulation and anti platelet medication. Minimally invasive techniques have been developed and modified over the years to now include endo-snaring, stapling, and sequential ligation [2022]. Atria Clipping is ideal for patients unable to undergo the Watchman's procedure. The most relevant challenge in the minimally-invasive approach to LAA closure has been achieving absolute occlusion of the LAA and, if possible, electrically isolating the appendage without tearing, associated bleeding, or damage to the left circumflex which falls near the LAA [23].

The improved success rate seen with the robotic approach to LAA closure can likely be attributed to the enhanced optics provided by the da Vinci three-dimensional endoscope [18]. However, as with all procedures, the surgical exclusion using a robotic approach does carry with it a documented risk. The main risk is the reopening of the LAA orifice, increasing the probability of clot formation and cardioembolic events [19]. Multiple trials show the efficacy of epicardial clipping of the LAA using a robotic approach. Our study confirms the available literature on the topic.

\section{Conclusion}

We believe that epicardial LAA clipping using a robotic approach is safe treatment option in high risk patients with $A F$ and is effective in physical and electrical exclusion of the LAA. It shows promising results, and we anticipate long term outcomes to be comparable to other techniques. Our robotic technique can decrease stroke risk and should be considered as a viable option in patients who have absolute contraindications for even short-term anticoagulation.

\section{References}

1. Benjamin EJ, Wolf PA, D'Agostino RB, et al. (1998) Impact of atrial fibrillation on the risk of death: The Framingham Heart Study. Circulation 98: 946-952.

2. Wolf PA, Kannel WB, McGee DL, et al. (1983) Duration of atrial fibrillation and imminence of stroke: The framingham study. Stroke 14: 664-667.

3. Blackshear JL, Odell JA (1996) Appendage obliteration to reduce stroke in cardiac surgical patients with atrial fibrillation. Ann Thorac Surg 61: 755-759. 
4. Wysowski DK, Nourjah P, Swartz L (2007) Bleeding complications with warfarin use: A prevalent adverse effect resulting in regulatory action. Arch Intern Med 167: 1414-1419.

5. Lip GY, Nieuwlaat R, Pisters R, et al. (2010) Refining clinical risk stratification for predicting stroke and thromboembolism in atrial fibrillation using a novel risk factor-based approach: The Euro heart survey on atrial fibrillation. Chest 137: 263-272.

6. Roldan V, Marin F, Fernandez H, et al. (2013) Predictive vale of the HAS-BLED and ATRIA bleeding scores for the risk of serious bleeding in a "real-world" population with atrial fibrillation receiving anticoagulant therapy. Chest 143: 179-184.

7. Aspberg S, Chang Y, Atterman A, et al. (2016) Comparison of the ATRIA, CHADS 2, and CHA 2 DS 2-VASc stroke risk scores in predicting ischaemic stroke in a large Swedish cohort of patients with atrial fibrillation. Eur Heart J 37: 3203-3210.

8. Pisters R, Lane DA, Nieuwlaat R, et al. (2010) A novel user-friendly score (HAS-BLED) to assess 1-year risk of major bleeding in patients with atrial fibrillation. Chest 138: 1093-1100.

9. Zhu W, He W, Guo L, et al. (2015) The HAS-BLED score for predicting major bleeding risk in anticoagulated patients with atrial fibrillation: A systematic review and meta-analysis. Clin Cardiol 38: 555-561.

10. Caldeira D, Costa J, Fernandes RM, et al. (2014) Performance of the HAS-BLED high bleeding-risk category, compared to ATRIA and HEMOR-R2HAGES in patients with atrial fibrillation: A systematic review and meta-analysis. J Interv Card Electrophysiol 40: $277-284$.

11. Madden JL (1949) Resection of the left auricular appendix; a prophylaxis for recurrent arterial emboli. J Am Med Assoc 140: 769-772.

12. Cox JL, Ad N, Palazzo T (1999) Impact of the maze procedure on the stroke rate in patients with atrial fibrillation. J Thorac Cardiovasc Surg 118: 833-840.

13. Kanderian AS, Gillinov AM, Pettersson GB, et al. (2008) Success of surgical left atrial appendage closure: Assessment by transesophageal echocardiography. J Am Coll Cardiol 52: 924-929.
14. Rosenzweig BP, Katz E, Kort S, et al. (2001) Thromboembolus from a ligated left atrial appendage. J Am Soc Echocardiogr 14: 396-398.

15. Ailawadi G, Gerdisch MW, Harvey RL, et al. (2011) Exclusion of the left atrial appendage with a novel device: Early results of a multicenter trial. J Thorac Cardiovasc Surg 142: 1002-1009.

16. Emmert MY, Puippe G, Baumuller S, et al. (2013) Safe, effective and durable epicardial left atrial appendage clip occlusion in patients with atrial fibrillation undergoing cardiac surgery: First long-term results from a prospective device trial. Eur J Cardiothorac Surg 45: 126-131.

17. Reddy VY, Sievert H, Halperin J, et al. (2014) Percutaneous left atrial appendage closure vs. warfarin for atrial fibrillation: A randomized clinical trial. JAMA 312: 1988-1998.

18. Ward AF, Applebaum RM, Toyoda N, et al. (2017) Totally endoscopic robotic left atrial appendage closure demonstrates high success rate. Innovations (Phila) 12: 46-49.

19. Lewis CTP, Stephens RL, Horst VD, et al. (2016) Application of an epicardial left atrial appendage occlusion device by a robotic-assisted, right chest approach. The Annals of Thoracic Surgery 101: e177-e178.

20. Bakhtiary F, Kleine P, Martens S, et al. (2008) Simplified technique for surgical ligation of the left atrial appendage in high-risk patients. J Thorac Cardiovasc Surg 135: 430-431.

21. Bartus K, Han FT, Bednarek J, et al. (2013) Percutaneous left atrial appendage suture ligation using the LARIAT device in patients with atrial fibrillation: Initial clinical experience. J Am Coll Cardiol 62: 108-118.

22. Blackshear JL, Johnson WD, Odell JA, et al. (2003) Thoracoscopic extracardiac obliteration of the left atrial appendage for stroke risk reduction in atrial fibrillation. J Am Coll Cardiol 42: 12491252.

23. Garcia FMA, Perez DA, Quiles J (2004) Role of left atrial appendage obliteration in stroke reduction in patients with mitral valve prosthesis: A transesophageal echocardiographic study. J Am Coll Cardiol 13: 60-61.

DOI: $10.36959 / 582 / 431$

Copyright: (c) 2021 Smith N, et al. This is an open-access article distributed under the terms of the Creative Commons Attribution License, which permits unrestricted use, distribution, and reproduction in any medium, provided the original author and source are credited. 